\title{
Longitudinal trajectories and predictors of adolescent suicidal ideation and attempts following inpatient hospitalization.
}

\section{Citation}

Prinstein, Mitchell J., Matthew K. Nock, Valerie Simon, Julie Wargo Aikins, Charissa S. L. Cheah, and Anthony Spirito. 2008. "Longitudinal Trajectories and Predictors of Adolescent Suicidal Ideation and Attempts Following Inpatient Hospitalization." Journal of Consulting and Clinical Psychology 76 (1): 92-103. doi:10.1037/0022-006x.76.1.92.

\section{Published Version}

doi:10.1037/0022-006X.76.1.92

\section{Permanent link}

http://nrs.harvard.edu/urn-3:HUL.InstRepos:33459450

\section{Terms of Use}

This article was downloaded from Harvard University's DASH repository, and is made available under the terms and conditions applicable to Open Access Policy Articles, as set forth at http:// nrs.harvard.edu/urn-3:HUL.InstRepos:dash.current.terms-of-use\#OAP

\section{Share Your Story}

The Harvard community has made this article openly available.

Please share how this access benefits you. Submit a story.

Accessibility 


\title{
Longitudinal Trajectories and Predictors of Adolescent Suicidal Ideation and Attempts Following Inpatient Hospitalization
}

\author{
Mitchell J. Prinstein, \\ Department of Psychology, University of North Carolina at Chapel Hill \\ Matthew K. Nock, \\ Department of Psychology, Harvard University \\ Valerie Simon, \\ Department of Psychology, Merrill Palmer Institute/Wayne State University \\ Julie Wargo Aikins, \\ Department of Psychology, University of Connecticut \\ Charissa S. L. Cheah, and \\ Department of Psychology, University of Maryland, Baltimore County \\ Anthony Spirito \\ Department of Psychiatry and Human Behavior, Warren Alpert Medical School of Brown \\ University
}

\begin{abstract}
Remarkably little is known regarding the temporal course of adolescent suicidal ideation and behavior, the prediction of suicidal attempts from changes in suicidal ideation, or the prediction of suicidal attempts after accounting for suicidal ideation as a predictor. A sample of 143 adolescents 12-15 years old was assessed during psychiatric inpatient hospitalization and again at 3, 6, 9, 15, and 18 months postdischarge through a series of structured interviews and parent- and adolescentreported instruments. Symptoms of depression, posttraumatic stress disorder, externalizing psychopathology, hopelessness, and engagement in several forms of self-injurious/suicidal behaviors (i.e., suicide threats/gestures, plans, nonsuicidal self-injury [NSSI]) were assessed. Latent growth curve analyses revealed a period of suicidal ideation remission between baseline and 6 months following discharge, as well as a subtle period of suicidal ideation reemergence between 9 and 18 months postdischarge. Changes in suicidal ideation predicted suicide attempts. After accounting for the effects of suicidal ideation, baseline suicide threats/gestures also predicted future suicide attempts. Higher adolescent-reported depressive symptoms, lower parentreported externalizing symptoms, and higher frequencies of NSSI predicted weaker suicidal ideation remission slopes. Findings underscore the need for more longitudinal research on the course of adolescent suicidality.
\end{abstract}

\section{Keywords}

suicidal ideation; suicidal attempts; nonsuicidal self injury; adolescence; longitudinal methods

\footnotetext{
Copyright 2008 by the American Psychological Association

Correspondence concerning this article should be addressed to: Mitchell J. Prinstein, University of North Carolina at Chapel Hill, Department of Psychology, Davie Hall, Campus Box 3270, Chapel Hill, NC 27599-3270. mitch.prinstein@ unc.edu.
} 
Recent data indicate that suicidal ideation, attempts, and completed suicide continue to occur at alarming rates, especially among youth (Kessler, Berglund, Borges, Nock, \& Wang, 2005; World Health Organization [WHO], 2005). In addition, epidemiological data suggest that the transition to adolescence represents a critical developmental vulnerability period for increased suicidal behavior. For instance, in the United States, suicide currently is the third leading cause of adolescent death, and the rate increases sixfold during the transition from childhood to adolescence (from 1.3 to 8.6 per 100,000; Centers for Disease Control and Prevention $[C D C], 2006)$. This developmental period also brings a dramatic increase in the occurrence of the immediate precursors to suicide death, including suicidal ideation, plans, and attempts (e.g., Kessler, Borges, \& Walters, 1999). National data from community-based samples of high school students indicate high rates of 12-month suicidal ideation (16.9\%), suicide plans $(13.0 \%)$, suicide attempts $(8.4 \%)$, and suicide attempts requiring medical treatment $(2.3 \%$; CDC, 2006) by adolescence. For these reasons, the study of suicidal behaviors at the transition stage to adolescence is of especially high priority (U.S. Department of Health and Human Services [HHS], 2000, 2001; U.S. Public Health Service, 1999; WHO, 2005).

Yet data bearing on some fundamental aspects of adolescent suicidal behavior are surprisingly unavailable. This is due in large part to the existence of only a small number of longitudinal investigations examining suicidal ideation or attempts over multiple time points. For instance, although previously cited data provide information about the lifetime and 12-month prevalence of suicidal behaviors, little is known about the temporal course of adolescent suicidal ideation or suicidal behavior. Prior longitudinal studies have offered some insight on the long-term course of suicidal ideation and attempts; however, the few studies that have done so often have assessed these outcomes by means of single-item indices or data collected at only two time points or at time points spaced quite far apart (e.g., 1-15 years between assessments; McKeown et al., 1998; Reinherz, Tanner, Berger, Beardslee, \& Fitzmaurice, 2006). Clinical experience suggests that fluctuations in suicidal behaviors occur much more rapidly. Thus, of particular scientific and clinical interest is the short-term course of suicidal ideation and behavior. For instance, it would useful to know whether suicidal ideation increases steadily over time among some adolescents, or whether ideation, like depressive episodes, may be cyclical in nature. Information such as this about the temporal course of suicidal ideation and attempts, while basic, would make a significant contribution to scientific and clinical efforts aimed at understanding and predicting these outcomes.

It is especially important to understand the course of suicidal ideation among high risk samples of youth with severe psychopathology, such as adolescent psychiatric inpatients. Suicidal ideation and attempts are a primary reason for referral for psychiatric hospitalization (Peterson, Zhang, Santa Lucia, King, \& Lewis, 1996), and although such thoughts and behaviors presumably decrease during hospitalization, they often reappear soon after discharge. The few studies that have focused on adolescents recently discharged from psychiatric hospitals have revealed that the greatest risk for suicide attempts occurs within 6 months to 1 year following hospital discharge; during this period, approximately 10\%-18\% of youth attempt suicide (Brent et al., 1993; Goldston et al., 1999; King et al., 1995; Spirito et al., 1992). Thus, although rates of suicidal ideation and attempts are elevated among youth, and prior data suggest risk of such outcomes are especially high among those recently discharged from psychiatric hospitalization, data are not currently available on the short-term course of suicidal ideation and attempts immediately following release from adolescent psychiatric hospitalization. Such information could significantly enhance our understanding of the nature of suicidal behaviors and also would offer essential information for clinical assessment and treatment planning. 
In addition to understanding the course of suicidal ideation and attempts, it also would be instructive to better understand the factors that predict changes in this course. Here, too, data are available from studies examining potential risk factors retrospectively at one time point (e.g., Kessler et al., 1999) or prospectively over extended periods of time (e.g., McKeown et al., 1998; Reinherz et al., 2006); however, studies of more proximal predictors of the presence of suicidal ideation and attempts are rare (Foley, Goldston, Costello, \& Angold, 2006). Moreover, few prior studies have been able to identify factors that are associated with suicide attempts after controlling for the effects of suicidal ideation. That is, most predictors of suicide attempts actually predict suicidal ideation but not which ideators will go on to make a suicide attempt (see Borges et al., 2006; Kessler et al., 1999). The identification of factors that can prospectively predict suicide attempts above and beyond prediction of suicidal ideation is important not only for clinical theory, but also for the clinical assessment and prevention of suicidality among youth.

Several different classes of predictors were considered on the basis of prior theoretical and empirical work in this area. First, we examined aspects of self-injury and suicidality that may portend a higher likelihood of future attempts. For instance, increases in suicidal ideation over time may prove useful in predicting short-term risk of suicide attempt. Clinicians often monitor the presence of suicidal ideation as a predictor of attempts, but the extent to which changes in ideation are associated with later attempts remains unclear. Additional indices of self-injury/suicidality — such as suicide plans, threats, or engagement in other self-injurious behaviors, even without suicidal intent (i.e., nonsuicidal self-injury [NSSI]; e.g., Nock, Joiner, Gordon, Lloyd-Richardson, \& Prinstein, 2006; Nock \& Prinstein, 2005) - also have been suspected as predictors of suicide attempts. This is consistent with recent theory suggesting that engagement in self-injurious behaviors or suicidality may produce habituation toward self-harm that contributes to one's capacity for engagement in more serious subsequent suicide attempts (Joiner, 2005). However, the extent to which these predictors are associated with subsequent suicide attempts after controlling for changes in suicidal ideation or past attempts has been surprisingly understudied.

Second, prior work has suggested that the presence of one or more psychiatric disorders is among the strongest predictors of suicidal ideation, attempts, and death (Brent et al., 1993; Foley et al., 2006; Nock \& Kessler, 2006; Shaffer et al., 1996). Most notably, affective and externalizing disorder symptoms are frequently noted as predictors of suicide attempts in past cross-sectional or retrospective studies, or as distal predictors of suicidal ideation and behavior (Brent et al., 1993; Kessler et al., 1999; Nock \& Kazdin, 2002; Nock \& Kessler, 2006; Shaffer et al., 1996). Evidence from long-term follow-up studies of hospitalized adolescents suggests that depressive symptoms, trait levels of hopelessness and anxiety, and symptoms of externalizing disorders may be associated with future attempts, even after controlling for past attempts (Goldston et al., 1999; Goldston, Reboussin, \& Daniel, 2006). The identification of psychological factors (both mental disorders and related psychological constructs) that are associated with short-term increases in suicidal ideation, and with increased risk of suicide attempt above and beyond the presence of suicidal ideation, would represent a significant clinical advance in this area.

The first goal of the current study was to prospectively examine the short-term trajectories of suicidal ideation and attempts among clinically referred adolescents in greater detail within this critical time period. Six repeated assessments were conducted within an 18month period following inpatient psychiatric hospitalization. It was hypothesized, on the basis of past work, that approximately $10 \%$ of adolescents would attempt suicide within 6 months of hospital discharge and 20\% within 18 months postdischarge (e.g., Goldston et al., 1999). It was expected that rates of suicidal ideation and attempts would be higher for girls than for boys at each time point, consistent with prior work on gender differences in the 
occurrence of these behaviors (Lewinsohn, Rohde, \& Seeley, 1996; Moscicki, 1999). It also was hypothesized that trajectories of suicidal ideation following inpatient hospitalization would not follow a simple linear trend, but instead would be characterized by periods of remission after hospital discharge, followed by a reemergence of ideation several months later, similar to the cyclical patterns that characterize depressive episodes. Some prior work provides a basis for this prediction by showing that declines in depressive symptoms predict subsequent declines in suicidal ideation among adults with major depressive disorder (Sokero et al., 2006). The current study represents an extension of this earlier finding and provides a more direct examination of the temporal pattern of adolescent suicidal ideation and attempts over time.

The second goal of the current study was to examine factors that might predict the occurrence of suicidal ideation and attempts among adolescents during the critical 18-month period following hospital discharge. First, it was hypothesized, on the basis of prior work in this area, that higher baseline levels of suicidal ideation, suicide attempts, and nonsuicidal self-injury would predict the subsequent occurrence of suicidal ideation and attempts. Second, it was hypothesized that if suicidal ideation and attempts do indeed fluctuate over the 18-month period following hospitalization (as proposed in the first study goal stated above), then changes in suicidal ideation over the 18-month period would predict subsequent changes in the risk of suicide attempts. That is, increases in suicidal ideation during one time period (i.e., slope of suicidal ideation) would predict an increased likelihood of suicide attempt during the next time period above and beyond earlier predictors. Third and finally, it was hypothesized that baseline psychological factors including internalizing disorders (e.g., depression, posttraumatic stress disorder), externalizing disorders (e.g., conduct and oppositional defiant disorders), and hopelessness would significantly predict the occurrence and trajectories of subsequent suicidal ideation and attempts.

\section{Method}

\section{Participants}

Participants included 143 adolescents ( $72 \%$ girls) between the ages of 12 and 15 years $(M=$ $13.51, S D=0.75)$ and in Grades $7(20 \%), 8(40 \%)$, or $9(40 \%)$ at baseline. This age range was chosen because of the significantly increased risk of suicidal thoughts and behaviors during this developmental period (Kessler et al., 1999). Approximately $75 \%$ of participants were White/Caucasian, 3\% African American, 4\% Latino American, and 17\% mixed ethnicity. Approximately $27 \%$ of adolescents lived with both biological parents, $29 \%$ with their biological mother only, and $15 \%$ with their biological mother and a stepparent; the remaining adolescents lived either with their biological father or extended family or in foster or temporary care. Based on maternal report, $19 \%$ of mothers had not obtained a high school diploma, $40 \%$ had a high school degree, $14 \%$ had earned a trade degree, $11 \%$ had some undergraduate college, and $16 \%$ had obtained a college degree or higher.

All participants were recruited from a psychiatric inpatient facility in the northeastern United States. During the period of recruitment, a total of 246 adolescents matching study inclusion (12-15 years old, no history of prior psychosis or mental retardation) were admitted to the inpatient unit. Both suicidal and non-suicidal adolescents were recruited to ensure variability in the constructs of interest for this investigation. At the time of this data collection, approximately $40 \%$ of all admissions onto this unit were discharged or transferred within 1 or 2 days of admission. This length of stay was associated with a variety of factors (e.g., insurance carrier, vacancies at local facilities) and did not serve as a marker for the severity of adolescents' psychological symptoms or socioeconomic status. Consistent with human subjects regulations, adolescents and their parents were approached for study participation after clinical personnel had met with adolescents' parent/guardian and gained 
permission for them to be approached about this investigation (i.e., typically on the second day following admission). Consent for study participation therefore was requested from 183 of these eligible adolescents. A total of 162 (88.5\%) provided consent, and $143(88.3 \%)$ of these were available to be assessed on study measures (i.e., 19 participants were discharged after consent was given but before data collection).

Adolescents and their parents initially were assessed during hospitalization (baseline) immediately following consent, typically within 2 to 4 days of admission. Adolescents and parents completed additional assessments at 3, 6, 9, 15, and 18 months post-baseline.

Data were missing for two potential reasons common to research of this type: First, logistical challenges with inpatient data collection (e.g., competing demands for patients' time, unexpected discharge or transfer) yielded missing data on some items or measures within participants. Second, data sometimes were missing because of attrition over the longitudinal interval (e.g., adolescents' relocation, study dropout, etc.). Many retention strategies were utilized, including frequent phone and mail contact with participants and their immediate and extended family members and friends, searches within public access databases for current contact information, and participant incentives for completion of follow-up assessments (i.e., $\$ 30$ at each follow-up time point for both a parent and the adolescent). Of the 143 adolescents who completed baseline assessments, a total of 133 (93\%) adolescents participated in at least one of the follow-up time points; $115(80 \%)$ participated in at least two follow-up time points, $106(74 \%)$ participated in at least three time follow-up points, 96 (67\%) in four follow-up time points, and $76(53 \%)$ completed every follow-up assessment. A total of 102 adolescents (71\%) participated at the final assessment. This retention rate is comparable to prior research. Boergers and Spirito (2003) reviewed 31 longitudinal follow-up studies of adolescent suicide ideators and attempters; only four conducted in the United States collected follow-up data for over a year. Retention rates for these four studies ranged from $52 \%$ to $83 \%$ (weighted $M=71 \%$ ).

Analyses were conducted to compare adolescents with or without complete longitudinal data on all baseline study variables. Analyses also were conducted to examine adolescents who did or did not participate in the final time point. In both cases, no significant effects were revealed on any study variables, suggesting no evidence for attrition biases. Missing data analyses indicated that data were missing at random, Little's MCAR $\chi^{2}(1840)=1,839.57$, $n s$. Because listwise deletion would unnecessarily omit valuable data, all analyses were conducted with all available data (see Data Analyses below). Analyses conducted on the basis of only available data revealed an identical pattern of results.

\section{Measures}

All adolescent questionnaire-based measures were read aloud by a trained research assistant during individual meetings while adolescents privately recorded their responses. This procedure allowed for adequate probing and explanation of study items when necessary, monitoring of adolescents' attention and conscientiousness while completing measures, and immediate checking for response inconsistencies.

Suicidality_Adolescents' suicidal ideation, suicide plans, suicide threats/gestures, and suicide attempts were assessed. The Suicidal Ideation Questionnaire (SIQ; W. M. Reynolds, 1985) was used to assess suicidal ideation. The SIQ includes 30 items designed to assess thoughts about suicide in adolescents. Items are scored on a 7-point scale ranging from 0 to 6 , with greater scores reflecting greater frequencies of suicidal ideation severity. The scales were developed through field testing with over 2,400 respondents. Construct validity of the SIQ has been demonstrated by correlations with highly related constructs such as depression $(r=.59)$ and hopelessness $(r=.48)$, as well as strong convergence with scores from a 
structured clinical interview regarding suicidal behavior (W. M. Reynolds, 1990). The SIQ was administered at all six time points; internal consistency was .92 or above at each time point. At baseline, suicidal ideation over the past 12 months was assessed; at each follow-up time point, ideation over the prior 3 months was assessed.

Items used to assess suicide plans, suicide threats/gestures, and suicide attempts were adapted from existing instruments designed to assess suicidal behavior, including the Kiddie-Schedule for Affective Disorders and Schizophrenia (Kaufman et al., 1997), the National Institute of Mental Health Diagnostic Interview Schedule for Children (NIMHDISC; Shaffer et al., 1993; 2000), and the Youth Risk Behavioral Surveillance System (Kann, Kolbe, \& Collins, 1993). Suicide plans were assessed by asking adolescents to indicate whether they had "made a plan about how you would attempt suicide?" Suicide threats/gestures were assessed by asking adolescents to report if they "tried to make someone believe that you might end your life, but didn't do it?" Suicide attempts were assessed by asking adolescents whether they "actually attempted suicide." At baseline, each construct was assessed to determine behavior over the past year. The lifetime history of suicide attempts also was assessed at baseline. At each subsequent time point, each construct was assessed to determine behavior within the 3 months from the prior assessment. The examination in this study of both suicidal ideation and attempts over multiple time points following hospitalization provided a novel opportunity to examine factors that might predict the individual baseline levels and temporal growth of suicidal ideation, and that predict suicide attempts over time, after accounting for initial suicidal ideation, growth in suicidal ideation, and past attempts.

Nonsuicidal self-injury-NSSI was assessed at baseline through two sets of items. An initial item examined the frequency within the past year that adolescents "harmed or hurt your body on purpose (for example, cutting or burning your skin, hitting yourself, or pulling out your hair) without wanting to die"; a 6 -point scale $(0=$ Never, $5=$ Once a day $)$ was used for this test. A second set of five items reported the frequency with which adolescents engaged in several types of nonsuicidal self-injurious behavior (i.e., cut/carved skin, hit self, pulled hair out, burned skin), without suicide intent, in the past year. The frequency of engagement in each item was reported on a 5 -point scale $(1=$ Never, $5=$ Almost every day $)$. A mean score across all five items was computed $(a=.70)$. The correlation between both sets of NSSI items was .69, $p<.001$.

Psychological symptoms-Several clinician-interview, self-report, and parent-report measures of psychological symptoms were administered at Time 1 to allow for the examination of latent variables within each construct assessed. The NIMH-DISC-IV (Shaffer et al., 2000) was used to obtain information regarding psychiatric diagnosis and psychological symptoms. Both adolescent (DISC-C) and parent (DISC-P) report versions were administered when possible; each is a structured interview developed for use concerning children and adolescents ages 6-17. Items assess symptoms, behaviors, and emotions corresponding to diagnostic criteria of the Diagnostic and Statistical Manual of Mental Disorders (4th ed.; DSM-IV; American Psychiatric Association, 1994). The NIMHDISC has demonstrated good to excellent diagnostic sensitivity (Fisher et al., 1993), interrater reliability (Shaffer et al., 1993, 2000), test-retest reliability (Jensen et al., 1995; Shaffer et al., 2000), and construct validity (Costello, Edelbrock, \& Costello, 1985; Weinstein, Noam, Grimes, Stone, \& Schwab-Stone, 1990). Symptoms of unipolar mood disorder (i.e., major depression module), posttraumatic stress disorder (PTSD), and externalizing behavior (oppositional defiant disorder and conduct disorder modules) were assessed. For each type of disorder, the number of adolescents' clinically significant symptoms was summed to produce a continuous measure reported by adolescents (internal consistency: major depression $=.90, \mathrm{PTSD}=.89$, conduct disorder $=.69)$ and their parents 
(internal consistency: major depression $=.92, \operatorname{PTSD}=.88$, conduct disorder $=.48$ ) at baseline.

Adolescents additionally completed the Children's Depression Inventory (CDI; Kovacs, 1992) as a measure of depressive symptoms at Time 1 . The CDI includes 27 items that assess cognitive and behavioral depressive symptoms, including all but one (psychomotor agitation) of the DSM-IV (American Psychiatric Association, 1994) criteria for a major depressive episode. A three-item response format, scored 0 through 2 , is used in which children endorse statements that best describe their level of depressive symptoms in the previous 2 weeks. A mean score was computed across all items with one exception (i.e., suicidal ideation) to minimize overlap between constructs; higher scores indicated higher levels of depressive symptoms. Good psychometric properties have been reported for the CDI as a reliable and valid index of depressive symptoms (Saylor, Finch, Spirito, \& Bennett, 1984); it can be used with youths between the ages of 7 and 18 years (Kazdin, 1990). Internal consistency in the present sample was .88 .

A self-reported measure of externalizing symptoms, the Delinquency Behavior Questionnaire, was adapted from the Self-Reported Delinquency Interview (Elliot, Huizinga, \& Ageton, 1985). Items assessed the frequency of adolescents' engagement in several illegal and aggressive behaviors frequently included in inventories of deviance and externalizing symptoms (e.g., engaging in a physical fight, vandalism, obscene phone calls, theft of personal property, arson, vehicular theft, weapon carrying, shoplifting, truancy). Adolescents reported their frequency of engaging in each behavior by means of a 5-point scale. Internal consistency for this measure was .88. A mean score across all items was correlated significantly with conduct disorder symptoms as reported on the NIMH-DISC-IV (Shaffer et al., 2000) by adolescents $(r=.78, p<.001)$ and by parents $(r=.29, p<.01)$.

Parents completed the Behavioral Assessment System for Children (BASC; C. R. Reynolds $\&$ Kamphaus, 1992). The Parent Report form, relating to adolescents ages 12-18 years, includes 126 items, each measured on a 4-point scale (i.e., Never, Sometimes, Often, Almost Always) to assess a variety of symptoms. Tscores for adolescents' parent-reported externalizing problems (i.e., including hyperactivity, aggression, conduct problems) and depression symptoms were used in analyses. Adequate internal consistency (as > .74) and 2to 8-week test-retest reliability (median $r=.70$ ) has been reported for each subscale of the BASC in community-based and clinically referred samples.

Hopelessness-Adolescents completed the Hopelessness Scale for Children (Kazdin, Rodgers, \& Colbus, 1986). This measure includes 17 true-false items designed to assess negative expectancies toward oneself and regarding the future. A total sum is computed (with reverse coding where appropriate) to generate an overall score of hopelessness symptoms ( $a=.90$ in this sample). Adequate internal consistency and validity have been demonstrated with adolescents (Spirito, Williams, Stark, \& Hart, 1988).

\section{Data Analysis}

Three sets of analyses were conducted to examine study hypotheses. Descriptive statistics first were conducted to examine the frequency of suicidal ideation and attempts over the 18month follow-up period. Because data were missing for some variables and time points, it is important to interpret the percentages rather than frequency counts in Table 1.

Second, it was of interest to better understand the course of suicidal ideation over the 18month longitudinal period. This was examined by constructing an unconditional growth curve model on the basis of latent curve analysis. The use of latent curves allowed for an estimation of the slope and pattern of growth within the entire sample, as well as predictors 
of individual temporal growth trajectories (Bollen \& Curran, 2006). An additional benefit of this approach is the ability to model individual intercepts and slopes when data are missing at random. All latent curve analyses were conducted with Amos 7.0 (Arbuckle, 1999). Means and intercepts were estimated on the basis of full information direct maximum likelihood when data were missing.

It was anticipated that suicidal ideation slopes might be nonlinear, given that for some adolescents ideation may be high at baseline (i.e., during hospitalization), lower following discharge, and possibly increasing again over the extended longitudinal period. An initial model examined a single latent slope factor. The six measures of suicidal ideation (SIQ scores at baseline, $3,6,9,15$, and 18 months postbaseline) were included as observed indicators, with intercepts set to 0 . A latent intercept factor with paths to all observed indicators set to 1 was modeled. Path weights between the latent slope factor and each observed indicator of suicidal ideation were allowed to vary freely with the exception of ideation at baseline (0) and 18 months (1), to allow for nonlinearity.

The single-slope model was compared with models examining (a) a piecewise approach (i.e., linear spline) or (b) a curvilinear-slope function. The use of the piecewise approach allowed for an examination of two separate slope functions (Bollen \& Curran, 2006). A first slope function modeled the curve between baseline, 3 , and 6 months postbaseline (i.e., an "ideation remission" curve). A second slope function modeled the curve between 9, 15, and 18 months postbaseline (i.e., an "ideation reemergence" curve). Growth curve modeling requires at least three time points to compute a slope. Each linear spline was modeled with two paths fixed (to 0 and 1, respectively) and the third path allowed to freely vary. The curvilinear model required the inclusion of an initial slope function (with paths to observed indicators set to indicate 3-month intervals: $0,1,2,3,5,6$, respectively), and a second slope function with each corresponding path weight squared (Bollen \& Curran, 2006).

The best fitting model from analyses above was built upon to examine the third set of hypotheses related to the prospective prediction of suicidal ideation and attempts. In addition to the prediction of suicidal ideation intercept and slope(s), three additional outcome variables were added reflecting (a) the presence/absence of a suicide attempt at baseline, (b) attempts between baseline and 9 months postbaseline, and (c) attempts between 9 and 18 months postbaseline. Paths initially were included to examine associations between each of these three indices of suicide attempts and to examine suicidal ideation intercepts and slopes as predictors of suicide attempts. Additional predictors then were entered into the model to predict suicidal ideation intercepts, suicidal ideation slopes, and suicide attempts above and beyond the effects of suicidal ideation on suicide attempts.

\section{Results \\ Descriptive Statistics}

Table 1 presents the means and standard deviations for all study variables, as well as the results of $t$ tests examining gender differences. Results indicated that just under half of the sample had attempted suicide prior to hospital admission. A total of 19 adolescents (13\% of the sample) reported suicide attempts within 6 months of hospital discharge. A total of 38 suicide attempts were reported by 33 adolescents (approximately $23 \%$ of the full sample) between hospital discharge and 18 months postbaseline ( 5 adolescents reported attempts at more than one follow-up time point). Chi-square analyses indicated that adolescents who reported a suicide attempt at baseline were significantly more likely than baseline nonattempters to report a suicide attempt at least once within 6 months of hospital discharge ( $24.4 \%$ of prior attempters vs. $3.9 \%$ of prior nonattempters), $\chi^{2}(1)=11.53, p<.01$, and at least once during the 18-month follow-up interval (36.4\% of prior attempters vs. $12.7 \%$ of 
prior nonattempters), $\chi^{2}(1)=10.50, p<.01$. Of the 33 adolescents who attempted suicide within 18 months postbaseline, $24(72.7 \%)$ were reattempters and $9(27.3 \%)$ were first time attempters.

Differences between these reattempters $(n=24)$ and first time attempters $(n=9)$ on the primary baseline variables included in this study were examined by means of $t$ tests. As would be expected from prior research, results indicated that reattempters reported higher levels of psychological symptoms than did adolescents who would later attempt suicide for the first time. On the NIMH-DISC-IV (Shaffer et al., 2000), this was evident for youthreported symptoms of depression: reattempters $(M=4.78, S D=3.49)$, first time attempters $(M=.63, S D=1.77), t(31)=4.33, p<.01$; generalized anxiety: reattempters $(M=2.83, S D$ $=2.71)$, first time attempters $(M=.62, S D=1.41), t(31)=2.92, p<.05$; PTSD: reattempters $(M=6.57, S D=6.57)$, first time attempters $(M=.50, S D=1.41), t(31)=4.16, p<.01$; and oppositional defiant disorder: reattempters $(M=3.09, S D=2.50)$, first time attempters $(M=$ $1.38, S D=1.30), t(31)=2.46, p<.05$. Significant differences also were revealed for baseline levels of NSSI: reattempters $(M=2.04, S D=0.78)$, first time attempters $(M=1.42$, $S D=0.47), t(31)=2.21, p<.05$; and depressive symptoms on the CDI: reattempters $(M=$ $0.96, S D=0.30)$, first time attempters $(M=0.75, S D=0.15), t(31)=2.70, p<.05$.

Analysis of gender differences indicated that girls reported higher levels of suicidal ideation at $6,9,15$, and 18 months postbaseline than did boys. Girls also were overrepresented in the proportion of adolescents who reported suicide attempts at baseline, but underrepresented in the proportion of adolescents who reported a suicide plan at baseline, as compared with boys. Girls reported significantly more episodes of NSSI at baseline, as well as greater severity of depression and anxiety symptoms, as compared with boys, on self-reported and youth-interview measures only (see Table 1).

\section{Course of Suicidal Ideation Over Time}

Analysis of unconditional growth curve models began with an examination of a one-slope model including baseline, 3-, 6-, 9-, 15-, and 18-month measures of suicidal ideation. The model was an adequate fit, $\chi^{2}(16)=22.66, p<.03 ; \chi^{2} / \mathrm{df}=1.89$; comparative fit index $(\mathrm{CFI})=.95$; root-mean-square error of approximation $(\mathrm{RMSEA})=.08$; Akaike information criterion $(\mathrm{AIC})=52.66$. This model was compared with a piecewise model with a first latent slope factor representing the slope between baseline (path weight $=0$ ), 3- (freely varying), and 6-month (1) time points (additional time point paths set to 1) and a second slope factor representing changes between 9- (freely varying), 15- (freely varying), and 18-month (1) time points (additional time point paths set to 0 ). This model also yielded a good fit, $\chi^{2}(5)=$ $13.96, n s ; \chi^{2} / \mathrm{df}=1.55 ; \mathrm{CFI}=.98 ; \mathrm{RMSEA}=.06 ; \mathrm{AIC}=49.69$. By setting all parameters, errors, and path weights for the second slope factor to zero and allowing estimation of paths between the first slope function and the latter three observed suicidal ideation indicators (i.e., to replicate the initial one-slope factor model above), it was possible to consider these two alternate models nested and reveal that the piecewise model was a statistically better fit to the data, $\Delta \chi^{2}(3)=8.7, p<.01$. In addition, a comparison of the AIC between the two models suggested that the piecewise model was better fit to the data than the one-slope factor model. A third model, with a quadratic slope factor, also was modeled. The fit for the quadratic model, $\chi^{2}(12)=31.35, p<01 ; \chi^{2} / \mathrm{df}=2.61 ; \mathrm{CFI}=.91 ; \mathrm{RMSEA}=.10 ; \mathrm{AIC}=$ 61.35 , was not statistically better than the initial one-slope model and fit significantly worse than the piecewise model, $\Delta \chi^{2}(3)=8.7, p<.0001$.

Thus, the piecewise model was used as a starting point for all analyses listed below. Constraining error variances across all six observed indicators of suicidal ideation did not significantly change model fit, $\chi^{2}(14)=15.97, n s ; \chi^{2} / \mathrm{df}=1.55 ; \mathrm{CFI}=.98 ; \mathrm{RMSEA}=.06$; $\mathrm{AIC}=41.69 ; \Delta \chi^{2}(5)=2.01, n s$, and thus constraints were retained for parsimony. The 
estimated unstandardized path weights for suicidal ideation at 3 months postbaseline on the first slope factor were $.82, p<.001$, and for suicidal ideation at 9 and 15 months postbaseline on the second slope factor were $.00, n s$, each. Estimated intercept parameters indicated that suicidal ideation was significantly greater than 0 at baseline $(M=2.99, p<$. $0001)$ with significant variability around this mean $(2.11, p<.05)$. Estimated parameters for the first slope factor $(M=-1.34, p<.001$; variance $=1.30, n s)$ indicate declining levels of suicidal ideation between baseline, 3 , and 6 months postbaseline (i.e., an ideation remission slope). Estimated parameters for the second slope factor indicate a marginally significant increasing slope in suicidal ideation between 9,15 , and 18 months postbaseline $(M=0.18, p$ $=.06$; variance $=68.00, n s$, i.e., an ideation reemergence slope) .

Gender differences were anticipated in the trajectories of suicidal ideation over time. A multiple group analysis was not possible, however, given a relatively small number of boys. Gender therefore was included as an exogenous predictor in analyses below.

\section{Suicidal Ideation Trajectories Predicting Suicide Attempts}

Three dichotomous outcome variables were added to the piecewise model described above to examine the prediction of suicide attempts. An exogenous indicator reflecting the presence or absence of suicide attempts at baseline was added, as well as two outcome variables reflecting (a) presence/absence of suicide attempts between baseline and 9 months postbaseline, and (b) presence/absence of suicide attempts between 9 and 18 months postbaseline (time points were combined to examine attempts by the halfway point and end point of follow-up, corresponding to the time points for the two slopes of suicidal ideation, and to increase cell sizes). Paths were estimated (a) between the suicidal ideation intercept and the three suicide attempt outcomes (i.e., suicide attempts at baseline, between baseline and 9 months, and between 9 and 18 months); (b) between the initial (i.e., ideation remission) slope factor with all three suicide attempt outcomes; and (c) between the second slope factor (i.e., ideation reemergence) and suicide attempts between 9 and 18 months postbaseline. Paths also were estimated between all three measures of suicide attempts. Model fit decreased notably, $\chi^{2}(26)=51.64, p<.01 ; \chi^{2} / \mathrm{df}=1.99 ; \mathrm{CFI}=.92 ; \mathrm{RMSEA}=$. $08 ; \mathrm{AIC}=107.64$. A nonsignificant path between suicide attempts by 9 and by 18 months postbaseline was trimmed, yielding a better model fit, $\chi^{2}(27)=28.30, n s ; \chi^{2} / \mathrm{df}=1.05$; CFI $=1.00 ;$ RMSEA $=.02 ; \mathrm{AIC}=82.30$.

In this model, the initial suicidal ideation intercept was associated significantly with a suicide attempt at baseline, $B=0.20, p<.001$, by 9 months postbaseline, $B=.22, p<.001$, and between 9 and 18 months postbaseline, $B=0.29, p<.001$, suggesting that higher baseline levels of suicidal ideation were associated with future attempts above and beyond the effects of initial attempts. The ideation remission slope was associated significantly with suicide attempts by 9 months postbaseline, $B=0.21, p<.05$, and between 9 and 18 months postbaseline, $B=0.31, p<.001$. The positive value of these regression weights coupled with the negative average slope value indicates weaker ideation remission slopes (i.e., approaching zero) were associated with a greater likelihood of future suicide attempts. Last, a significant association was revealed between the ideation reemergence slope and suicide attempts between 9 and 18 months postbaseline, $B=0.23, p<.05$, indicating that steeper increasing slopes of ideation reemergence were associated with a greater likelihood of future attempts. An additional significant path was revealed between suicide attempts by 9 months, and between 9 and 18 months postbaseline, $B=-0.18, p<.05$, reflecting that attempters at 9 months were unlikely to attempt again before 18 months, and attempters at 18 months were unlikely to have attempted at 9 months. Interestingly, once the association between suicidal ideation trajectories and suicide attempts was accounted for, a significant association between initial suicide attempts and future attempts no longer remained significant, $B=$ $0.14, n s$. 


\section{Baseline Self-Injury and Suicidality as Predictors of Suicidal Ideation Trajectories and Suicide Attempts}

The next goal of analyses was to examine predictors of suicidal ideation trajectories and predictors of suicide attempts above and beyond suicidal ideation intercepts and slopes as predictors. An initial model examined self-injury predictors at baseline, as well as gender. Specifically, four predictors were added to the model listed above: a suicide plan at baseline; a suicide threat/gesture at baseline; the frequency of NSSI at baseline (modeled as a latent factor, with both observed measures of NSSI as indicators); and gender. Paths between each of these predictors and (a) the suicidal ideation intercept, (b) both suicidal ideation slopes, and (c) all three measures of suicide attempts were estimated; all predictors were allowed to covary. The fit of this model was adequate, $\chi^{2}(50)=94.36, p<.001 ; \chi^{2} / \mathrm{df}=1.89 ; \mathrm{CFI}=$. 92; RMSEA = .08; AIC $=232.36$. All regression weights are listed in Table 2. As might be expected, several associations among baseline measures were revealed. The presence of a suicide plan and more episodes of NSSI each were associated with higher levels of baseline suicidal ideation (i.e., intercept). Above and beyond the association between suicidal ideation intercepts and baseline attempts (which remained significant), and correlations with other self-injury predictors, the presence of a suicide plan also was associated with a greater likelihood of baseline suicide attempts.

Results also revealed several interesting longitudinal associations between baseline suicidality and self-injury with later suicidal ideation and attempts. After all other estimated associations were accounted for, higher frequencies of NSSI were associated with lower suicidal ideation remission slopes (i.e., slopes approaching zero). The presence of a suicide threat/gesture also was associated with a greater likelihood of suicide attempts between 9 and 18 months postbaseline.

\section{Psychological Symptoms as Predictors of Suicidal Ideation Trajectories and Suicide Attempts}

A final goal was to examine the association between baseline psychological symptoms, measured by clinician interview, adolescent and parent reports, and trajectories of suicidal ideation and future suicide attempts. Initial analyses revealed that latent factors of psychological symptom domains including both adolescent- and parent-reported data did not fit the data well. Thus, separate analyses were conducted for adolescent- and for parentreported data.

Analysis of adolescent-reported data included two latent factors and three observed indicators as predictors of (a) suicidal ideation intercept and slopes, and (b) all three measures of suicide attempts, as in the model above. The two latent factors included depression (two indicators: adolescent-reported NIMH-DISC-IV depression symptoms and CDI scores) and externalizing symptoms (three indicators: adolescent-reported NIMHDISC-IV oppositional defiant disorder and conduct disorder symptoms and well as Delinquent Behavior Questionnaire scores). Observed indicators included NIMH-DISC-IV PTSD symptoms, adolescents' scores on the Hopelessness Scale for Children, and adolescents' gender. The model fit the data well, $\chi^{2}(82)=116.46, p<.01 ; \chi^{2} / \mathrm{df}=1.42$; $\mathrm{CFI}=.95$; RMSEA $=.05 ; \mathrm{AIC}=292.46$. Results are presented in Table 3 . Results suggested that only adolescent-reported depressive symptoms were associated with higher initial levels of suicidal ideation (i.e., intercept) and lower declining slopes of suicidal ideation in the first 9 months postdischarge (i.e., remission slopes approaching zero).

An identical model was conducted to examine adolescents' parent-reported symptoms as predictors of suicidal ideation trajectories and suicide attempt outcomes. Latent depression and externalizing factors were constructed on the basis of results from the NIMH-DISC-IV's 
parent interview (depression symptoms, and oppositional defiant disorder and conduct disorder symptoms, respectively) and scores on the BASC Depression and Externalizing Problems subscales. PTSD scores on the parent-reported NIMH-DISC-IV also were included. The model fit the data adequately, $\chi^{2}(76)=122.32, p<.01 ; \chi^{2} / \mathrm{df}=1.61 ; \mathrm{CFI}=$. 91; RMSEA $=.06 ;$ AIC $=274.32$. Results are presented in Table 4. Significant effects were revealed only for externalizing symptoms; higher levels of externalizing symptoms were associated with lower levels of initial suicidal ideation and greater remission slopes (i.e., quicker recovery).

\section{Discussion}

Although decades of excellent research have been dedicated toward understanding the phenomenology and predictors of suicidality, remarkably little is known regarding some basic questions about suicide. Among these understudied issues are the course of suicidal ideation and behavior over time, the short-term prediction of suicide attempts from recent changes in suicidal ideation, and the predictors (i.e., both self-injurious behaviors and psychological symptoms) that are associated with suicide attempts above and beyond suicidal ideation. This study addressed each of these issues within a population of youth that is at greatest risk for suicide (i.e., psychiatrically hospitalized inpatients) at the critical developmental period that is associated with unique vulnerabilities to suicide attempts and completions.

An initial goal of this study was to better understand the course of suicidal ideation and behavior. A few prior studies have examined the prevalence of attempts among adolescents following discharge from inpatient psychiatric facilities (e.g., Brent et al., 1993; Goldston et al., 1999; King et al., 1995; Spirito et al., 1992). Our data offered an important replication of these findings: Between one fifth and one quarter of adolescent inpatients attempted suicide within 18 months after discharge, and the vast majority of these attempters had attempted suicide prior to their hospital admission. Girls also were more likely than boys to attempt suicide following hospital discharge. The $13.9 \%$ reattempt rate in the 3 months following discharge appears slightly higher than the $10 \%$ rate found at the same hospital almost 15 years earlier (Spirito et al., 1992). Our findings offer an important corroboration of past work and highlight the importance of focusing intervention and prevention efforts on adolescents with a previous suicide attempt who are recently discharged from a psychiatric hospital. Adolescents must be closely monitored following hospitalization, given the extremely high risk during this short period. Perhaps the greatest obstacle to suicide prevention efforts is the low rate at which suicidal behaviors occur and the associated difficulties that this introduces to prediction (Cohen, 1986; Goldstein, Black, Nasrallah, \& Winokur, 1991). This problem would be partially addressed if prevention efforts focused more intensively on high risk individuals during this critical period for suicide attempts.

This study also closely studied trajectories of suicidal ideation following inpatient hospitalization. Most prior studies of suicidal ideation and attempts have examined the presence versus absence of these phenomena and have revealed less about the parameters of suicidal experiences, such as their duration or reoccurrence. Notably, studies in which parameters of suicidal phenomena have been examined have provided useful information about how suicidal thoughts and attempts differ over time (e.g., Joiner \& Rudd, 2000; Joiner, Rudd, Rouleau \& Wagner, 2000). Adding to this literature, our findings suggested that the average course of ideation in this sample included both a period of substantial decline in suicidal ideation during the first 6 months following hospitalization, followed by a subtle reemergence of ideation in the year that followed. Findings suggest that suicidal ideation, like depressive symptoms, may follow a cyclical course. Although practitioners may regard the passing of time after an acute crisis or attempt as suggesting a gradually 
decreasing risk of future ideation, these results suggest that the reemergence of suicidal thoughts may be a relatively common phenomenon within this population. Moreover, over time and across repeated suicide attempts, it may require less stress to precipitate a suicidal crisis (e.g., Joiner \& Rudd, 2000). Ongoing monitoring of suicidal ideation is critical for many months following psychiatric hospitalization, and perhaps particularly following a latency period of initial declines and apparent ideation remission. Unfortunately, many of these adolescents are no longer in treatment a year after hospital discharge and therefore are not able to be routinely monitored by a trained professional (Spirito, Boergers, Donaldson, Bishop, \& Lewander, 2002). Effective prevention of suicide will likely require monitoring of at risk adolescents more broadly and may be enhanced by consideration of warning signs for suicide (e.g., Rudd et al., 2006).

Although in this sample a reemergence of suicidal ideation was revealed between 6 and 18 months following hospital discharge, it should not be concluded that this specific time period will be consistent among adolescents or across samples. Our analytic strategy influenced the number of time points included in estimation of each slope factor.

Examination of means suggested that elevations may have occurred most notably between 15 and 18 months following discharge. It also is important to note that even at the lowest time point (i.e., 15 months), ideation remained present to some degree, with a mean of 1.53 on a $0-6$ scale. This suggests that although variation in mean level of ideation was observed, the results also suggest some chronicity of ideation among adolescents following discharge from the hospital. Moreover, although variability around the mean slope coefficients was not statistically significant, individual differences nevertheless will be present in the timing of suicidal ideation reemergence. Nevertheless, this study offers novel and important data on the short-term course of suicidal ideation following hospitalization and suggests the presence of ongoing risk for later ideation and attempts.

It will be especially important to understand factors other than time since hospital discharge that might be significantly associated with later increases in suicidal ideation or behavior. Thus, a second set of study goals involved the examination of baseline factors that would predict later suicidal ideation trajectories and suicide attempts. Consistent with past research, results suggested that initial levels of suicidal ideation were associated with greater risks of future suicide attempts. However, and particularly important, results also suggested that changes in suicidal ideation over time also were significantly associated with future attempts, even after accounting for the role of baseline ideation and attempts as predictors. In other words, not only is the identification of past suicidal thoughts or behavior important for predicting future suicidal behavior, but the rate at which adolescents' suicidal ideation abates following discharge, and the rate at which ideation reemerges, uniquely contribute toward understanding future risks. In fact, once these trajectories of suicidal ideation were accounted for, a past suicide attempt was no longer a significant predictor of later attempts. An important direction for future work on suicide is the development of more complex models that use temporal changes in suicidal ideation and other risk factors in the prediction of suicide attempts. Most prior work has tested potential risk factors measured at one point in time in the prediction of suicide attempts at some point in the future. This is a fairly blunt approach to risk assessment, and the current findings suggest that consideration of shortterm changes in factors such as suicidal ideation may lead to better predictive models than what is possible by means of even the strongest static predictors.

Results also suggested longitudinal associations between specific self-injurious/suicidal behaviors and later suicide attempts that were statistically significant even after accounting for (a) initial levels of ideation and (b) trajectories of suicidal ideation as predictors. Higher frequencies of NSSI episodes also were associated with weaker ideation remission following hospital discharge. Broadly, results are consistent with Joiner's (2005) recent theory 
regarding the habituation effects of self-injurious nonattempts escalating toward engagement in a suicide attempt. It is possible that engagement in NSSI lowers the threshold of stress required to precipitate a self-injurious or suicidal episode (cf. Pettit, Joiner \& Rudd, 2004). Moreover, consistent with Nock and Prinstein (2005), the association between NSSI and persisting suicidal ideation may be due to functional similarities between these behaviors; both may be manifestations of a general desire to stop aversive stimuli, such as social pressures. The exploration of functional similarities between various self-injurious behaviors is another high priority for future research.

Beyond the associations among suicidal ideation, self-injurious behaviors, and suicide attempts, surprisingly few unique effects were revealed for adolescent- or parent-reported symptoms as predictors of suicidality. Higher levels of adolescent-reported depressive symptoms were associated with weaker ideation remission, while higher levels of parentreported externalizing symptoms were associated with less baseline ideation and stronger remission slopes. Perhaps most notable about these results is the absence of more significant effects. For the prediction of suicidal ideation, results suggest that, beyond the initial effect of suicidal ideation on later ideation trajectories, baseline externalizing, PTSD, and hopelessness symptoms do not offer a unique contribution toward understanding ideation. Gender also was not significantly associated with trajectories after accounting for initial ideation. For the prediction of suicide attempts, results suggest that some psychological symptoms may exert only an indirect influence on suicidal behavior via the trajectories of suicidal ideation. Adolescent-reported symptoms of baseline depression and parent-reported externalizing symptoms at baseline are associated with the course of recovery following hospitalization; however, these factors do not help to identify the occurrence of suicide attempts following hospital discharge.

Overall, results demonstrated that trajectories of suicidal ideation over time are an important and potent factor to consider in understanding the risk for suicide attempts following hospital discharge. A history of suicidal gestures/threats before hospitalization also may suggest a unique risk for posthospitalization attempts. Beyond these factors, however, little is known regarding baseline factors that predict later attempts, after accounting for suicidal ideation trajectories. Clearly, this is an extremely high priority for future research. Moreover, it will be critical also to examine time-varying factors that are associated with both suicidal ideation trajectories and suicide attempts posthospitalization.

Future research also should address the limitations of this study. First, the generality of these findings is limited by the adolescent psychiatric inpatient sample used. It will be important not only to replicate these findings, but to test these effects with older adolescents and more diverse samples. Second, although the sample size was adequate to test our primary hypotheses, we lacked the statistical power to conduct tests that were more fine-grained, such as different predictive models for different genders and ethnic groups. As mentioned above, the accurate prediction of suicide attempts will require increasingly complex predictive models, and these will necessitate increasingly larger sample sizes. Third, while the time frame we examined was shorter than in prior large-scale studies, it is likely that many of the constructs examined (e.g., suicidal ideation, affective disorder symptoms) change much more rapidly than in 3-month segments. Therefore, it will be important in future research to examine even shorter windows of time in the prediction of suicidal outcomes. Fourth, the range of predictors included in this study was necessarily limited. Suicidal thought and behaviors are multidetermined outcomes, and while it was important to remain focused in the current study, given our sample size, it will be important to begin to integrate findings across studies to develop more inclusive models in order to better predict and prevent these dangerous behaviors. 
Overall, this study of suicidal ideation trajectories reveals what may be a common course of symptoms occurring within the 18 months following discharge from a psychiatric inpatient facility among youth at the critical developmental period associated with suicidal behavior and completions. Results offered specific directions for a new generation of research that specifically examines short-term fluctuations in suicidal ideation that may be predictive of attempts only a few months later. The longitudinal examination of adolescent suicidality remains an extremely high research priority.

\section{Acknowledgments}

This work was supported in part by grants from the National Institute of Mental Health (R01-MH59766) and the American Foundation for Suicide Prevention awarded to Mitchell J. Prinstein. Special thanks are due to Li Cai for his extremely helpful statistical consultation.

\section{References}

American Psychiatric Association. Diagnostic and statistical manual of mental disorders. 4. Washington, DC: Author; 1994.

Arbuckle, JL. Amos (Version 7.0) [Computer software]. Chicago: Smallwaters; 1999.

Boergers, J.; Spirito, A. Follow-up studies of child and adolescent suicide attempters. In: King, RA.; Apter, A., editors. Suicide in children and adolescents. New York: Cambridge University Press; 2003. p. 271-293.

Bollen, KA.; Curran, PJ. Latent curve models: A structural equation perspective. Hoboken, NJ: Wiley; 2006.

Borges G, Angst J, Nock MK, Ruscio AM, Walters EE, Kessler RC. A risk index for 12-month suicide attempts in the National Comorbidity Survey Replication (NCS-R). Psychological Medicine. 2006; 36(12):1747-1757. [PubMed: 16938149]

Brent DA, Perper JA, Moritz G, Allman C, Friend A, Roth C, et al. Psychiatric risk factors for adolescent suicide: A case-control study. Journal of the American Academy of Child and Adolescent Psychiatry. 1993; 32(3):521-529. [PubMed: 8496115]

Centers for Disease Control and Prevention. Youth Risk Behavior Surveillance-United States, 2005. Morbidity and Mortality Weekly Report: Surveillance Summary. 2006; 55(SS-5):1-108.

Cohen J. Statistical approaches to suicidal risk factor analysis. Annals of the New York Academy of Sciences. 1986; 487(1):34-41. [PubMed: 3471164]

Costello EJ, Edelbrock CS, Costello AJ. Validity of the NIMH Diagnostic Interview Schedule for Children: A comparison between psychiatric and pediatric referrals. Journal of Abnormal Child Psychology. 1985; 13(4):579-595. [PubMed: 4078188]

Elliot, DZ.; Huizinga, D.; Ageton, SS. Explaining delinquency and drug use. Beverly Hills, CA: Sage; 1985.

Fisher PW, Shaffer D, Piacentini JC, Lapkin J, Kafantaris V, Leonard H, et al. Sensitivity of the Diagnostic Interview Schedule for Children, 2nd edition (DISC-2.1) for specific diagnoses of children and adolescents. Journal of the American Academy of Child and Adolescent Psychiatry. 1993; 32(3):666-673. [PubMed: 8496131]

Foley DL, Goldston DB, Costello EJ, Angold A. Proximal psychiatric risk factors for suicidality in youth: The Great Smoky Mountains Study. Archives of General Psychiatry. 2006; 63(9):10171024. [PubMed: 16953004]

Goldstein RB, Black DW, Nasrallah A, Winokur G. The prediction of suicide: Sensitivity, specificity, and predictive value of a multivariate model applied to suicide among 1,906 patients with affective disorders. Archives of General Psychiatry. 1991; 48(5):418-422. [PubMed: 2021294]

Goldston DB, Daniel SS, Reboussin DM, Reboussin BA, Frazier PH, Kelley AE. Suicide attempts among formerly hospitalized adolescents: A prospective naturalistic study of risk during the first 5 years after discharge. Journal of the American Academy of Child and Adolescent Psychiatry. 1999; 38:660-671. [PubMed: 10361783] 
Goldston DB, Reboussin BA, Daniel SS. Predictors of suicide attempts: State and trait components. Journal of Abnormal Psychology. 2006; 115:842-849. [PubMed: 17100542]

Jensen P, Roper M, Fisher P, Piacentini J, Canino G, Richters J, et al. Test-retest reliability of the Diagnostic Interview Schedule for Children (DISC 2.1): Parent, child, and combined algorithms. Archives of General Psychiatry. 1995; 52(1):61-71. [PubMed: 7811163]

Joiner, TE. Why people die by suicide. Cambridge, MA: Harvard University Press; 2005.

Joiner TE, Rudd MD. Intensity and duration of suicidal crises vary as a function of previous suicide attempts and negative life events. Journal of Consulting and Clinical Psychology. 2000; 68(5): 909-916. [PubMed: 11068977]

Joiner TE Jr, Rudd MD, Rouleau MR, Wagner KD. Parameters of suicidal crises vary as a function of previous suicide attempts in youth inpatients. Journal of the American Academy of Child and Adolescent Psychiatry. 2000; 39(7):876-880. [PubMed: 10892229]

Kann L, Kolbe L, Collins J. Measuring the health behavior of adolescents: The Youth Risk Behavior Surveillance System and recent reports on high-risk adolescents. Public Health Reports. 1993; 108(Suppl 1):1-67.

Kaufman J, Birmaher B, Brent D, Ran U, Flynn C, Moreci P, et al. Schedule for affective disorders and schizophrenia for school-age children — present and lifetime version (KSADS-PL): Reliability and validity. Journal of the American Academy of Child \& Adolescent Psychiatry. 1997; 36:980988. [PubMed: 9204677]

Kazdin, AE. Assessment of childhood depression. In: La Greca, AM., editor. Through the eyes of the child: Obtaining self-reports from children and adolescents. Needham Heights, MA: Allyn \& Bacon; 1990. p. 189-233.

Kazdin AE, Rodgers A, Colbus D. The Hopelessness Scale for Children: Psychometric characteristics and concurrent validity. Journal of Consulting and Clinical Psychology. 1986; 54:241-245. [PubMed: 3700812]

Kessler RC, Berglund P, Borges G, Nock MK, Wang PS. Trends in suicide ideation, plans, gestures, and attempts in the United States, 1990-1992 to 2001-2003. Journal of the American Medical Association. 2005; 293(20):2487-2495. [PubMed: 15914749]

Kessler RC, Borges G, Walters EE. Prevalence of and risk factors for lifetime suicide attempts in the National Comorbidity Survey. Archives of General Psychiatry. 1999; 56(7):617-626. [PubMed: 10401507]

King C, Segal H, Kaminski K, Naylor M, Ghaziuddin N, Radpour L. A prospective study of adolescent suicidal behavior following hospitalization. Suicide and Life Threatening Behavior. 1995; 25:327-338. [PubMed: 8553413]

Kovacs, M. Children's Depression Inventory Manual. New York: Multi-Health Systems; 1992.

Lewinsohn PM, Rohde P, Seeley JR. Adolescent suicide ideation and attempts: Prevalence, risk factors, and clinical implications. Clinical Psychology: Science and Practice. 1996; 3:25-46.

McKeown RE, Garrison CZ, Cuffe SP, Waller JL, Jackson KL, Addy CL. Incidence and predictors of suicidal behaviors in a longitudinal sample of young adolescents. Journal of the American Academy of Child and Adolescent Psychiatry. 1998; 37(6):612-619. [PubMed: 9628081]

Moscicki, EK. Epidemiology of suicide. In: Jacobs, DG., editor. The Harvard Medical School guide to suicide assessment and intervention. San Francisco: Jossey-Bass; 1999. p. 40-51.

Nock MK, Joiner TE Jr, Gordon KH, Lloyd-Richardson E, Prinstein MJ. Non-suicidal self-injury among adolescents: Diagnostic correlates and relation to suicide attempts. Psychiatry Research. 2006; 144(1):65-72. [PubMed: 16887199]

Nock MK, Kazdin AE. Examination of affective, cognitive, and behavioral factors and suicide-related outcomes in children and young adolescents. Journal of Clinical Child and Adolescent Psychology. 2002; 31(1):48-58. [PubMed: 11845650]

Nock MK, Kessler RC. Prevalence of and risk factors for suicide attempts versus suicide gestures: Analysis of the National Comorbidity Survey. Journal of Abnormal Psychology. 2006; 115(3): 616-623. [PubMed: 16866602]

Nock MK, Prinstein MJ. Clinical features and behavioral functions of adolescent self-mutilation. Journal of Abnormal Psychology. 2005; 114(1):140-146. [PubMed: 15709820] 
Peterson BS, Zhang H, Santa Lucia R, King RA, Lewis M. Risk factors for presenting problems in child psychiatric emergencies. Journal of the American Academy of Child and Adolescent Psychiatry. 1996; 35(9):1162-1173. [PubMed: 8824060]

Pettit JW, Joiner TE Jr, Rudd MD. Kindling and behavioral sensitization: Are they relevant to recurrent suicide attempts? Journal of Affective Disorders. 2004; 83(2-3):249-252. [PubMed: 15555722]

Reinherz HZ, Tanner JL, Berger SR, Beardslee WR, Fitzmaurice GM. Adolescent suicidal ideation as predictive of psychopathology, suicidal behavior, and compromised functioning at age 30 . American Journal of Psychiatry. 2006; 163(7):1226-1232. [PubMed: 16816228]

Reynolds, CR.; Kamphaus, RW. Behavior assessment system for children. Circle Pines, MN: American Guidance Service; 1992.

Reynolds, WM. Suicidal Ideation Questionnaire. Odessa, FL: Psychological Assessment Resources; 1985.

Reynolds WM. Development of a semistructured clinical interview for suicidal behaviors in adolescents. Psychological Assessment. 1990; 2:382-390.

Rudd MD, Berman AL, Joiner TE Jr, Nock MK, Silverman MM, Mandrusiak M, et al. Warning signs for suicide: Theory, research, and clinical applications. Suicide and Life Threatening Behavior. 2006; 36(3):255-262. [PubMed: 16805653]

Saylor C, Finch AJ, Spirito A, Bennett B. The Children's Depression Inventory: A systematic evaluation of psychometric properties. Journal of Consulting and Clinical Psychology. 1984; 52:955-967. [PubMed: 6520288]

Shaffer D, Fisher P, Lucas CP, Dulcan MK, Schwab-Stone ME. NIMH Diagnostic Interview Schedule for Children Version IV (NIMH DISC-IV): Description, differences from previous versions, and reliability of some common diagnoses. Journal of the American Academy of Child \& Adolescent Psychiatry. 2000; 39(1):28-38. [PubMed: 10638065]

Shaffer D, Gould MS, Fisher P, Trautman P, Moreau D, Kleinman M, et al. Psychiatric diagnosis in child and adolescent suicide. Archives of General Psychiatry. 1996; 53(4):339-348. [PubMed: 8634012]

Shaffer D, Schwab-Stone M, Fisher P, Cohen P, Piacentini J, Davies M, et al. The Diagnostic Interview Schedule for Children-Revised Version (DISC-R): I. Preparation, field testing, interrater reliability, and acceptability. Journal of the American Academy of Child \& Adolescent Psychiatry. 1993; 32(3):643-650. [PubMed: 8496128]

Sokero P, Eerola M, Rytsälä H, Melartin T, Leskelä U, Lestelä-Mielonen P, et al. Decline in suicidal ideation among patients with MDD is preceded by decline in depression and hopelessness. Journal of Affective Disorders. 2006; 95(1-3):95-102. [PubMed: 16780959]

Spirito A, Boergers J, Donaldson D, Bishop D, Lewander W. An intervention trial to improve adherence to community treatment by adolescents after a suicide attempt. Journal of the American Academy of Child and Adolescent Psychiatry. 2002; 41:435-442. [PubMed: 11931600]

Spirito A, Plummer B, Gispert M, Levy S, Kurkjian J, Lewander W, et al. Follow-up outcome of adolescent suicide attempters. American Journal of Orthopsychiatry. 1992; 62:464-468. [PubMed: 1497112]

Spirito A, Williams C, Stark L, Hart K. The Hopelessness Scale for Children: Psychometric properties with normal and emotionally disturbed adolescents. Journal of Abnormal Child Psychology. 1988; 16:445-458. [PubMed: 3221033]

U.S. Department of Health and Human Services. Healthy People 2010. Washington, DC: U.S. Government Printing Office; 2000.

U.S. Department of Health and Human Services. National strategy for suicide prevention: Goals and objectives for action. Rockville, MD: Author; 2001.

U.S. Public Health Service. The surgeon general's call to action to prevent suicide. Washington, DC: U.S. Government Printing Office; 1999.

Weinstein SR, Noam GG, Grimes K, Stone K, Schwab-Stone M. Convergence of DSM-III diagnoses and self-reported symptoms in child and adolescent inpatients. Journal of the American Academy of Child \& Adolescent Psychiatry. 1990; 29(4):627-634. [PubMed: 2387799] 
World Health Organization. Suicide prevention. 2005. Retrieved October 26, 2005, from http:// www.who.int/mental_health/prevention/suicide/suicideprevent/en/ 
Table 1

Descriptive Statistics for Primary Study Variables and Tests of Gender Differences

\begin{tabular}{|c|c|c|c|c|}
\hline Variable & Total & Boys & Girls & Statistic \\
\hline \multicolumn{5}{|l|}{ Suicidal ideation, $M(S D)$} \\
\hline Baseline & $2.99(1.73)$ & $2.63(1.81)$ & $3.14(1.68)$ & $t(141)=1.59$ \\
\hline 3 months & $1.91(1.17)$ & $1.65(1.19)$ & $2.02(1.15)$ & $t(117)=1.57$ \\
\hline 6 months & $1.72(1.01)$ & $1.34(0.48)$ & $1.89(1.13)$ & $t(104.87)^{a}=3.49^{* * *}$ \\
\hline 9 months & $1.76(1.11)$ & $1.34(0.75)$ & $1.93(1.18)$ & $t(78)^{a}=2.97^{*}$ \\
\hline 15 months & $1.53(0.88)$ & $1.21(0.27)$ & $1.66(1.01)$ & $t(91.03)^{a}=3.49^{* *}$ \\
\hline 18 months & $1.82(1.23)$ & $1.31(0.54)$ & $2.03(1.37)$ & $t(99.87)^{a}=3.83^{* *}$ \\
\hline \multicolumn{5}{|c|}{ Suicide attempts, $n(\%)$ reporting attempt } \\
\hline Baseline (past year) & $66(47.5 \%)$ & $13(32.5 \%)$ & $53(53.5 \%)$ & $\chi^{2}(1)=5.06^{*}$ \\
\hline 3 months $(n=115)$ & $16(13.9 \%)$ & $3(8.6 \%)$ & $13(16.3 \%)$ & $\chi^{2}(1)=1.12, n s$ \\
\hline 6 months $(n=105)$ & $3(2.9 \%)$ & 0 & $3(4.2 \%)$ & \\
\hline 9 months $(n=100)$ & $10(10.0 \%)$ & $2(6.7 \%)$ & $8(11.4 \%)$ & $\chi^{2}(1)=0.53, n s$ \\
\hline 15 months $(n=101)$ & $3(3.0 \%)$ & 0 & $3(4.2 \%)$ & \\
\hline 18 months $(n=101)$ & $6(5.9 \%)$ & 0 & $6(8.5 \%)$ & \\
\hline \multicolumn{5}{|c|}{ Other baseline suicide behaviors, $n(\%)$} \\
\hline Suicide plan & $75(53.6 \%)$ & $27(36.0 \%)$ & $48(64.0 \%)$ & $\chi^{2}(1)=4.37^{*}$ \\
\hline Threat/gesture $b$ & $77(74.8 \%)$ & $24(31.2 \%)$ & $53(68.8 \%)$ & $\chi^{2}(1)=0.11, n s$ \\
\hline \multicolumn{5}{|l|}{ Nonsuicidal self-injury, $M(S D)$} \\
\hline Overall frequency & $1.48(1.64)$ & $0.91(1.56)$ & $1.75(1.61)$ & $t(138)=2.29^{*}$ \\
\hline Frequency of specific behaviors & $1.54(0.62)$ & $1.35(0.57)$ & $1.61(0.63)$ & $t(138)=2.59^{*}$ \\
\hline \multicolumn{5}{|c|}{ Baseline psychological symptoms reported by adolescents, $M(S D)^{\mathcal{C}}$} \\
\hline Major depression & $2.53(3.02)$ & $1.16(2.27)$ & $3.06(3.12)$ & $t(91.97)^{a}=3.93^{* *}$ \\
\hline Social phobia & $1.40(1.95)$ & $0.89(1.62)$ & $1.60(2.03)$ & $t(83.67)^{a}=2.12^{*}$ \\
\hline Generalized anxiety & $1.19(2.13)$ & $0.58(1.43)$ & $1.43(2.31)$ & $t(108.17)^{a}=2.58^{*}$ \\
\hline PTSD & $3.29(5.29)$ & $1.82(4.01)$ & $3.87(5.63)$ & $t(94.24)^{a}=2.38^{*}$ \\
\hline Oppositional defiant & $2.09(2.25)$ & $1.42(2.05)$ & $2.35(2.28)$ & $t(134)=2.18^{*}$ \\
\hline Conduct disorder & $1.16(2.09)$ & $1.22(2.21)$ & $1.13(2.05)$ & $t(134)=0.20, n s$ \\
\hline \multicolumn{5}{|c|}{ Baseline psychological symptoms reported by parents, $M(S D)^{d}$} \\
\hline Major depression & $2.93(3.26)$ & $2.44(3.13)$ & $3.14(3.31)$ & $t(112)=1.04, n s$ \\
\hline Social phobia & $1.68(1.97)$ & $1.59(1.97)$ & $1.72(1.99)$ & $t(112)=0.33, n s$ \\
\hline Generalized anxiety & $1.08(2.17)$ & $0.85(1.74)$ & $1.18(2.33)$ & $t(112)=0.73, n s$ \\
\hline PTSD & $1.48(3.65)$ & $1.09(2.67)$ & $3.97(2.59)$ & $t(112)=0.75, n s$ \\
\hline Oppositional defiant & $4.24(2.64)$ & $4.88(2.67)$ & $3.96(2.59)$ & $t(112)=-1.72, n s$ \\
\hline Conduct disorder & $1.73(2.01)$ & $2.09(2.11)$ & $1.57(1.97)$ & $t(112)=-1.26, n s$ \\
\hline \multicolumn{5}{|l|}{ Other baseline symptoms, $M(S D)$} \\
\hline Depression (CDI) & $0.72(0.36)$ & $0.63(0.37)$ & $0.76(0.35)$ & $t(141)=2.01^{*}$ \\
\hline
\end{tabular}




\begin{tabular}{lcccc}
\hline Variable & Total & Boys & Girls & Statistic \\
\hline Social anxiety & $2.55(1.01)$ & $2.36(0.95)$ & $2.63(1.02)$ & $t(141)=1.38$ \\
Anxiety & $1.38(0.56)$ & $1.29(0.55)$ & $1.42(0.57)$ & $t(141)=1.15$ \\
Delinquency (DBQ) & $1.77(0.75)$ & $1.91(0.82)$ & $1.72(0.71)$ & $t(141)=-1.34$ \\
BASC depression $T$ & $78.93(18.26)$ & $77.35(14.94)$ & $79.43(19.27)$ & $t(81)=-0.44$ \\
BASC externalizing $T$ & $73.06(19.25)$ & $77.94(16.86)$ & $71.65(19.79)$ & $t(81)=1.23$ \\
Hopelessness (HSC) & $0.68(0.29)$ & $0.72(0.26)$ & $0.66(0.30)$ & $t(141)=-0.95$ \\
\hline
\end{tabular}

Note. PTSD = posttraumatic stress disorder; CDI = Children's Depression Inventory (Kovacs, 1992); DBQ = Delinquency Behavior Questionnaire; BASC $=$ Behavioral Assessment System for Children (C. R. Reynolds \& Kamphaus, 1992); HSC = Hopelessness Scale for Children (Kazdin, Rodgers, \& Colbus, 1986).

a Equal variances not assumed.

${ }^{b}$ Suicide threats/gestures were not assessed for the first 22 participants enrolled in the study.

${ }^{c}$ A total of 135 adolescents completed the Youth Report of the National Institute of Mental Health Diagnostic Interview Schedule for ChildrenFourth Edition (NIMH-DISC-IV; Shaffer et al., 2000).

${ }^{d}$ A total of 113 parents completed the Parent Report of the NIMH-DISC-IV.

*

$p<.05$.

**

$p<.01$. 


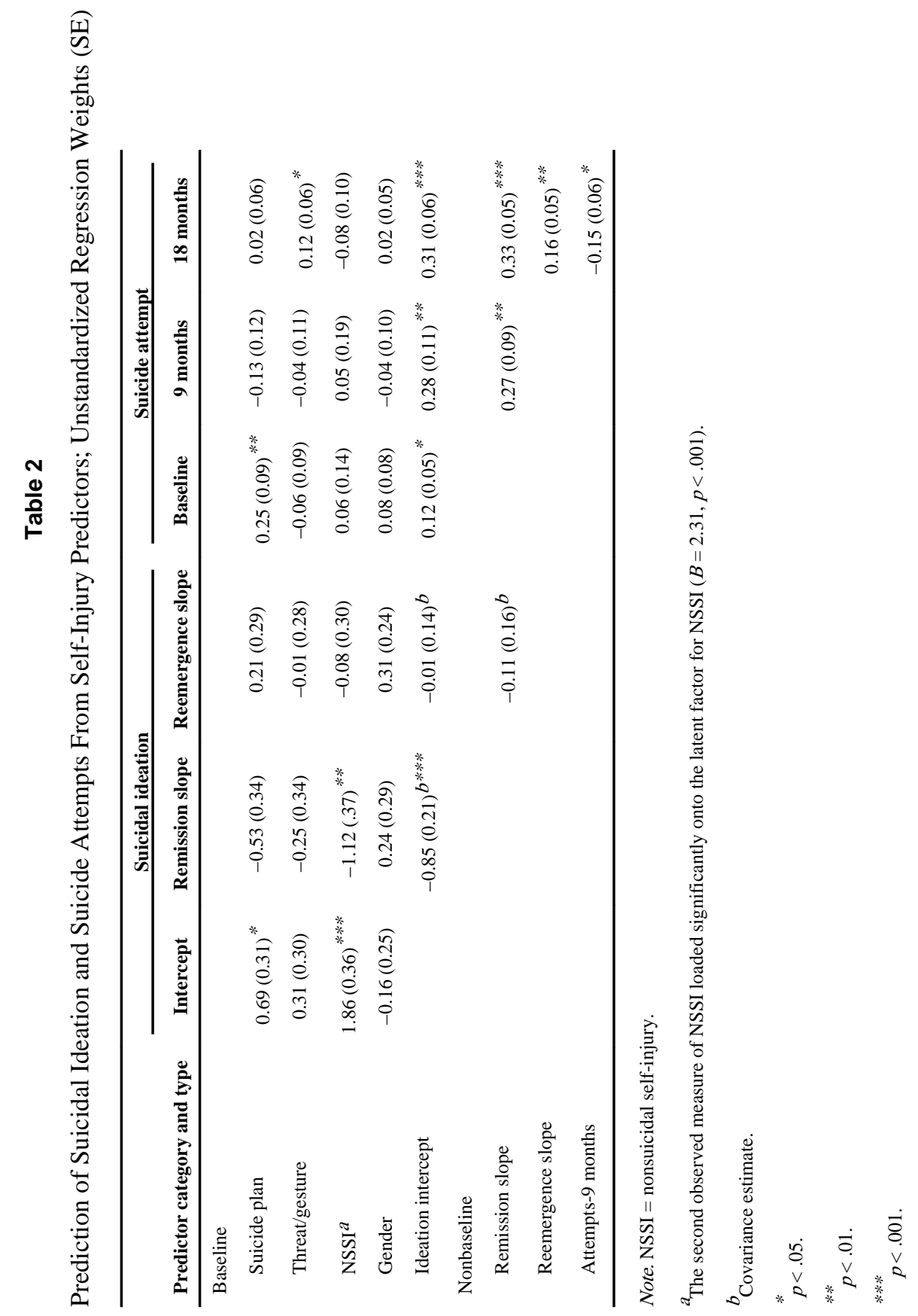




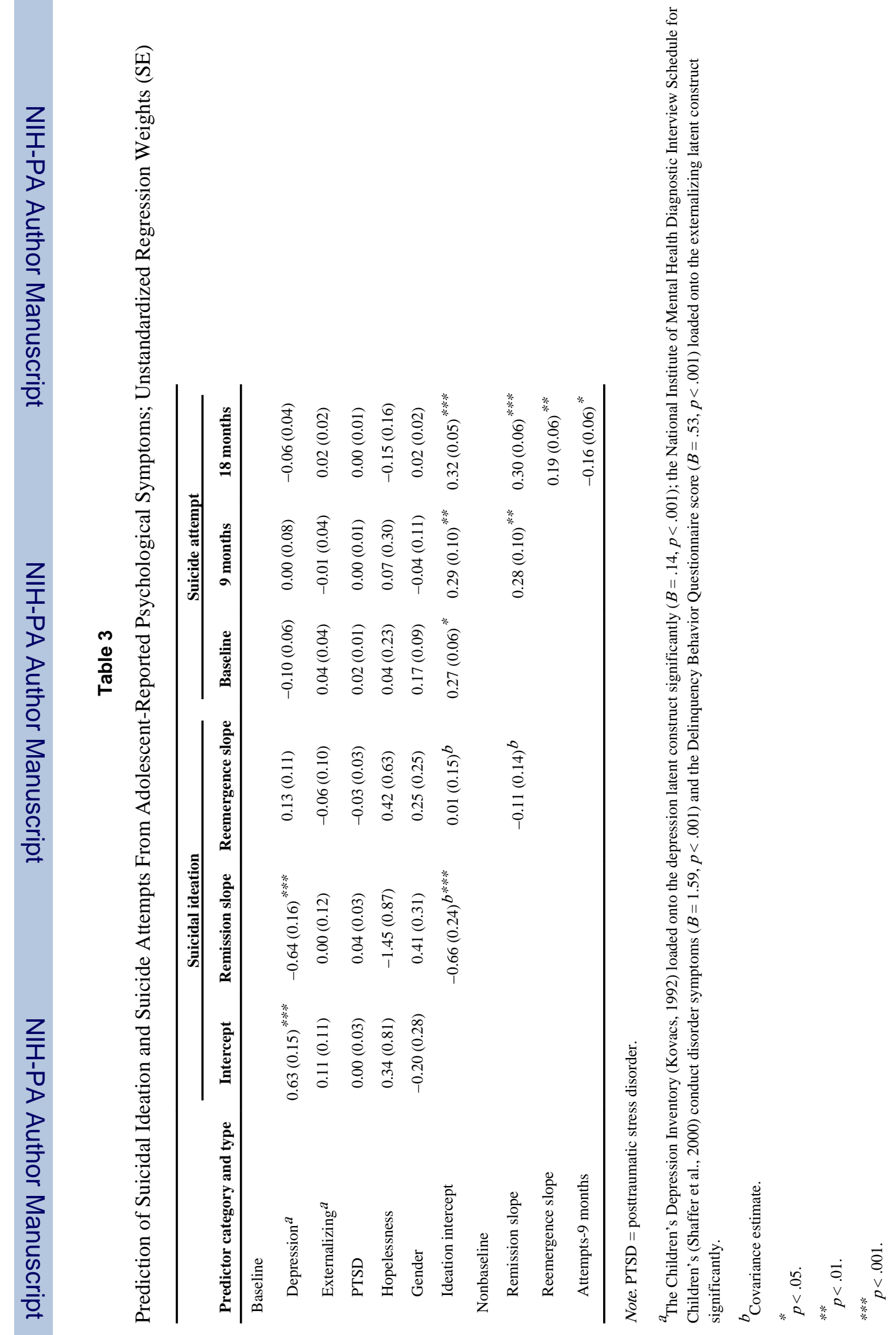

J Consult Clin Psychol. Author manuscript; available in PMC 2013 July 08. 


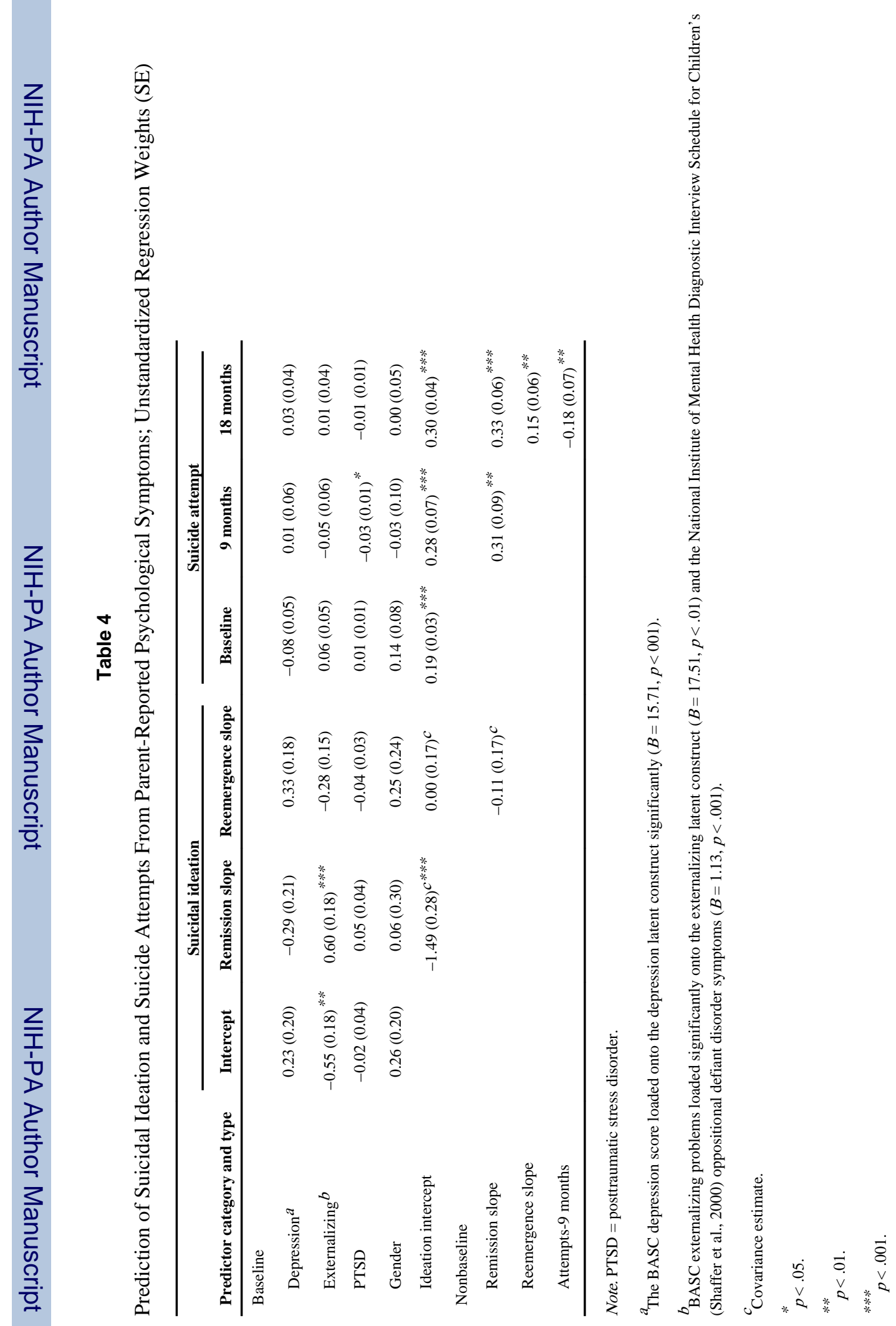

J Consult Clin Psychol. Author manuscript; available in PMC 2013 July 08. 